\title{
Paper Optimization of Direct Direction Finding Method with Two-Dimensional Correlative Processing of Spatial Signal
}

\author{
Vitaliy V. Tsyporenko and Valentyn G. Tsyporenko \\ Zhytomyr State Technological University, Zhytomyr, Ukraine
}

https://doi.org/10.26636/jtit.2018.115417

\begin{abstract}
In this article, the main parameter of the correlative-interferometric direction finding method with twodimensional correlative processing of spatial signal in the aperture of a linear antenna array (AA) is determined as the value of spatial shift within the $\mathrm{AA}$ aperture. The corresponding objective function is also formed. Analytical optimization of this parameter is presented and a comparative analysis of analytical calculations based on simulation results is conducted. In the simulation, a range of dependencies of the middle square deviation of estimation of direction on the value of the spatial shift for a signal-to-noise ratio of $0 \mathrm{~dB}$, for minimum 3-sample and 4-sample Blackman-Harris windows of the spectral analysis, is received. The value of the middle square deviation of estimation of direction will be minimal and will equal 0.02 degrees using a minimum 3-sample Blackman-Harris window with the $-67 \mathrm{~dB}$ level of side lobes. It offers high noise immunity and high accuracy of direction finding.
\end{abstract}

Keywords-antenna array, correlative interferometric, direct method, radio direction finding.

\section{Introduction}

Modern radio electronic telecommunications and radio monitoring systems must function in a difficult and rapidly changing electromagnetic situation (EMS). The said situation is characterized by multipath radio propagation, frequency coverage of the desired signal, as well as by intrusive noises and an a priori uncertainty. It is to because of a large number of mobile network devices operating in dense urban areas. With these conditions taken into consideration, the use of an adaptive digital antenna array (AA) is a promising area of improvement for telecommunications and radio monitoring systems. Its application allows to increase functional efficiency of telecommunications and radio monitoring systems by significantly optimizing AA parameters and operating modes. Optimization of the configuration of AA, the number and location of elements within its aperture, their number, methods for processing the weight of AA element signals, the form and the relationship between parameters of the main and the side lobes of the AA radiation pattern, are widely used methods. Dif- ferent criteria of optimization are presented in this paper. Also, at the expense of preliminary estimation of directions of interference sources, zeros in the radiation pattern (RP) of a digital adaptive AA can be formed. Optimization of the means of estimation of EMS allows to reduce the cost of telecommunications and radio monitoring equipment and significantly increases the speed of such systems.

The adaptation speed of a digital AA and its interference immunity depend significantly on the time-intensity and computational costs of the current EMS estimation methods, on the correlation interference matrix and on identification directions towards its source. Therefore, the task of increasing the speed of EMS analysis and estimating directions towards the sources of noise in a digital AA is relevant. The promising direction for the implementation of direction finding (DF) for these conditions is the use of digital wide-band correlative-interferometric direction finders coupled with AA, and the digital synthesis of its radiation pattern [1], [2].

Correlation-interferometric DF techniques offer a wide frequency range, resistance to interference caused by multipath reception, high sensitivity and good accuracy. However, the most plausible and unbiased assessment of estimation of directions towards radio emission sources (RES) is based on the search of a sequential correlational analysis and on the review of space. This greatly limits performance and requires a lot of hardware-related expenditure for data processing systems, therefore reducing the effectiveness of their application in dynamic EMS conditions [1]. Another disadvantage of this method is the low DF precision of RES, with the spectra being completely overlapped by frequency.

Effectiveness of DF depends on such parameters as speed, accuracy, noise immunity and hardware quality. Therefore, research on and optimization of digital direct correlativeinterferometric direction finders, as well as their adaptation to specific EMS conditions, are an important scientific challenge.

In [3], the direct method of DF with two-dimensional correlative processing of a spatial signal reconstructed in the aperture of a linear antenna array which provides a combination of high speed and precision direction finding, was 
presented. However, this method has not been researched and optimized under real life conditions yet.

In [4], [5] correlative-interferometric methods of estimating directions towards RES, using AA, which are effectively implemented in a digital domain, were studied. However, they rely on the search compensatory method of DF, which is slow in wideband DF of RES.

In [6], [7] the results of optimizing radio signal processing algorithms and basic parameters of digital correlation direction finders that rely on different AA configurations were obtained. The high level of efficiency in monitoring noise-like radio signals in a complex EMS was shown.

In [8]-[10] nonlinear spectral methods of DF that provide a high level of spatial resolution of the received signal were studied. However, they have a number of significant drawbacks for radio monitoring systems, such as:

- high computational complexity, being several times higher than that of the searching correlative DF method,

- requirement of a priori information on the exact amount of emission received in the mix,

- biased direction estimates which reduce DF accuracy,

- loss of operational stability at low signal-to-noise levels (about $10 \mathrm{~dB}$ ).

As a result, the efficiency of spectral DF methods which is determined, in the first place, by the speed-to-accuracy ratio, is insufficient in radio monitoring systems. This is confirmed by the absence of their application in modern digital DF of radio monitoring systems [1], [2].

In [11]-[14], the effectiveness and optimization of primary methods used for estimating direction towards RES, using AA, is researched. The optimal algorithms used for estimation of spatial radio emission parameters, as well as the optimal relationships between radiation pattern (RP) parameters ensuring effective spatial selection were determined. However, in these articles, optimization of the direct methods of correlation-interferometric DF, including the use of two-dimensional reconstructed signal processing, have not been investigated. Therefore, the results obtained there cannot be directly used to solve the research problem in question.

In light of the above, optimization, development and analysis of digital methods of correlation-interferometric DF to be applied in automated radio monitoring systems continue to be task with a high degree of urgency.

The aim of the article is to research and optimize the direct digital method of correlative-interferometric radio DF with two-dimensional correlative processing of spatial signal.

\section{Analytical Approach}

First, optimization of the direct digital method of correlative-interferometric DF with two-dimensional correlative processing of spatial signal reconstructed in the linear
AA aperture [3] to provide a minimum DF variance error is performed. Let a horizontal linear equidistant AA, consisting of $Z$ identical DF radio channels, receive a useful random Gaussian stationary signal $S(t)$ from an unknown direction $\theta$. It has a uniform energy spectrum $S^{2}(\omega)$, the width of which does not exceed the width $\Delta f_{k}$ of the band of DF radio channels. Radio channels of the AA have their own additive stationary normal noise $n_{z}(t)$, with a zero mathematical expectation and an equal two-sided spectral density $N$ of power, constant within the passband $\left[\omega_{S . L} ; \omega_{S, . H}\right]$ of the radio direction finder. We assume that the own noises of radio channels of the AA are not characterized by inter-channel correlation and correlation with the signal. Also, we assume that the phase fluctuations at the signal paths are absent. Thus, the initial research conditions are:

$$
U_{z}(t)=S_{z}\left(t-\tau_{z}\right)+n_{z}(t),
$$

where:

- $U_{z}(t)$ is the mixture taken by the $z$-th DF channel,

- $S_{z}\left(t-\tau_{z}\right)$ is the useful signal received by the $z$-th DF channel,

- $\tau_{z}$ is the delay of reception of the desired signal by the $z$-th DF channel, relative to the reference channel, depending on the direction towards RES,

- $n_{z}(t)$ is the own additive Gaussian noise within the band of the simultaneous analysis of the $z$-th DF channel.

DF should be performed within time $T_{a}$ of the analysis process, and within one correlation processing cycle, to ensure that the conditions correspond to the DF method analyzed, estimation $\hat{\theta}$ of direction towards RES is [3]:

$$
\hat{\theta}=\arccos \frac{\hat{\Omega}}{\omega_{S, L}},
$$

where $\hat{\Omega}=\frac{1}{\Delta z} \arctan \frac{\sum_{k=k_{l}}^{k_{H}} U_{v k}\left(\Omega_{p}\right) \sin \left[\Delta \hat{\Psi}_{A k}\left(\Delta z, \Omega_{p}\right)\right]}{\sum_{k=k_{l}}^{k_{H}} U_{v k}\left(\Omega_{p}\right) \cos \left[\Delta \hat{\Psi}_{A k}\left(\Delta z, \Omega_{p}\right)\right]}$

is the estimation of the spatial frequency of the signal group for received signal $S(t)$ using dispersion-correlation processing:

- $c$ is the propagation speed of electromagnetic emission in space,

- $\Delta z$ is the spatial shift within the AA aperture.

The $U_{v k}\left(\Omega_{p}\right)$ is defined, in above equation, by:

$$
\begin{aligned}
U_{v k}\left(\Omega_{p}\right)=\left\{\left[\sum_{p=p_{L}}^{p_{H}} U_{k}^{2}\left(\Omega_{p}\right) \sin \left(\Omega_{p} \Delta z\right)\right]^{2}\right. \\
\left.+\left[\sum_{p=p_{L}}^{p_{H}} U_{k}^{2}\left(\Omega_{p}\right) \cos \left(\Omega_{p} \Delta z\right)\right]^{2}\right\}^{\frac{1}{2}} .
\end{aligned}
$$


$U_{v k}\left(\Omega_{p}\right)$ is the module of the complex mutual space (obtained by implementing spatial processing) spectrum of the $k$-th frequency component of emission $S(t)$ received by the linear AA with multi-lobe RP. The other designations are:

- $\Omega_{p}=\frac{2 \pi p}{d Z}$ is the value of spatial frequency, which determines the direction of the $p$-th lobe of the multilobe RP, $p=0,1, \ldots, Z-1$,

- $p_{L}, p_{H}$ are the numbers of lower and upper spatial frequencies of the selected signal group,

- $d$ is the distance between AA elements,

- $\Delta \hat{\Psi}_{A k}\left(\Delta z, \Omega_{p}\right)=\arctan \frac{\sum_{p=p_{L}}^{p_{H}} U_{k}^{2}\left(\Omega_{p}\right) \sin \left(\Omega_{p} \Delta z\right)}{\sum_{p=p_{L}}^{p_{H}} U_{k}^{2}\left(\Omega_{p}\right) \cos \left(\Omega_{p} \Delta z\right)}$

is the estimation of the phase shift spatial of analytic signal in the distance,

- $\omega_{S . L}, \omega_{S . k}$ are the lower and the $k$-th frequencies of spectrum $U_{z}\left(\mathrm{j} \omega_{S . k}\right)$ of the received mixture $U_{z}(t)$, respectively,

- $k_{L}, k_{H}$ are the numbers of lower and upper frequencies of the spectrum $U_{z}\left(\mathrm{j} \omega_{S . k}\right)$ of the received mixture $U_{z}(t)$, respectively.

Analysis of Eq. (2) shows that the estimation $\hat{\theta}$ of the direction towards RES is determined on the basis of twodimensional space-time correlation processing of a complex mutual spatial spectrum $U_{v k}\left(\Omega_{p}\right)$ of the emission $S(t)$ received from the linear AA, with multi-lobe RP within the time $T_{a}$ of the analysis. Counts of the mutual spatial spectrum $U_{v k}\left(\Omega_{p}\right)$ are determined by spatial correlation processing of the selected signal group. Thus, the $k$-th signal group is a complex array $\left\{U_{k}\left(\mathrm{j} \Omega_{p}\right)\right\}_{p \in\left[p_{L} ; p_{H}\right]}$ of spatial spectrum components from the AA, with the multi-lobe RP on action of the $k$-th time spectral component with frequency $\omega_{S . k}$ for emission, received by overlapping main lobes of adjacent partial RP.

The use of parallel frequency and spatial analysis with the following dispersion-correlation estimation of direction allows to obtain the direct estimation $\hat{\theta}$ of direction towards RES.

The variant of the direction finder structure, implementing the analyzed method is shown in Fig. 1.

The direction finder shown in Fig. 1 is characterized by the following features. The mixture of $U_{z}(t)$ of radio emission is received by the $Z$-channel linear AA. The first channel of the AA is constantly connected to the first input of a twochannel radioreceiver with a common heterodyne. The AA channels from 2 to $Z$ are connected to the second input of the radio receiver, using an antenna switch, at the time $T_{a}$ of the analysis. From the two intermediate frequency outputs of the radio receiver, the pairs of signals are formed by a two-channel analog-to-digital converter (ADC) and are converted into a digital form with a sampling frequency
$F_{d}=2 \Delta f_{k}$. As a result, $Z-1$ pairs of arrays on $N_{s}=T_{a} F_{d}$ samples of the received mixture $U_{z}(t)$ of radio emission are accumulated and transmitted to the computing unit. Then, using the fast Fourier transform (FFT) algorithm, $Z-1$ pairs of signal spectra are obtained. From the received pairs of spectra the reciprocal spectra are formed by means of a complex conjugation of the spectrum of the first channel AA and its multiplication with signals from 2 to $Z$ channel AA that are equivalent to the spectra of signals obtained by using the $Z$ channel radio receiver.

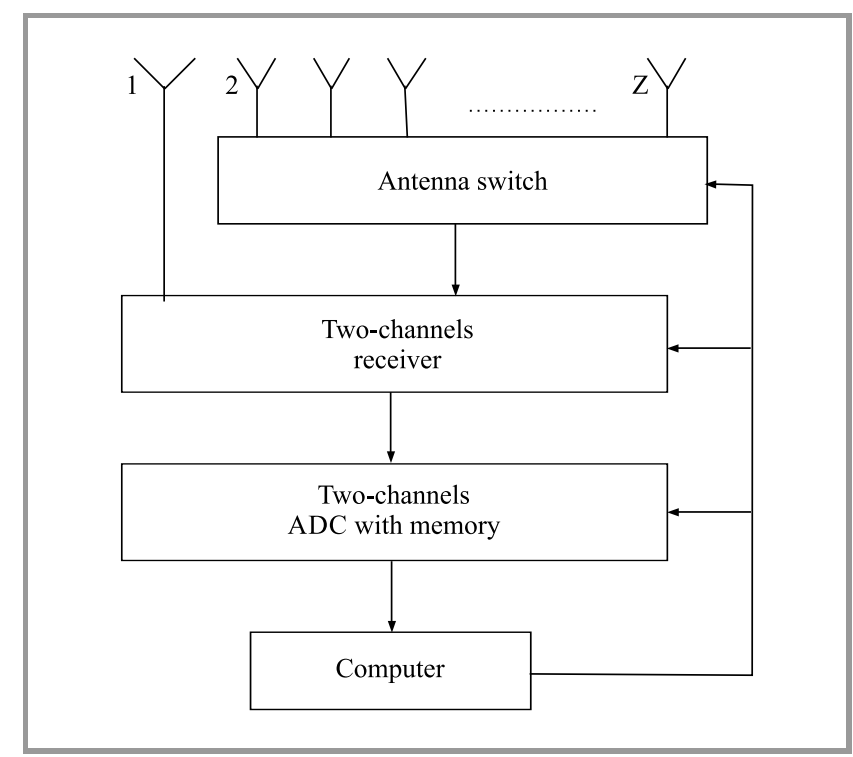

Fig. 1. Structure scheme of correlative-interferometric DF with two-dimensional correlative processing of spatial signal.

According to the proposed DF method [3], subsequent processing of the received mutual spectra is performed on a computer, based on Eq. (2). The use of a two-channel radio receiver, compared to the -channel minimizes hardware costs, but leads to an increase in the total time of radio emission analysis by $Z-1$ times.

The operation of the antenna switch, of the radio receiver and of the ADC is controlled by a computer.

The main indicator of the accuracy of DF is dispersion $\sigma_{\theta}^{2}$ of the direction estimation error. It is determined, in the DF method analyzed, according to [2], [11]:

$$
\begin{aligned}
\sigma_{\theta}^{2}=\frac{2 K_{W \theta} c^{2}}{m_{s}} & \\
& \times \frac{1}{\omega^{2} T_{a} \mu_{I} \Delta f_{k} Z} K_{\tau}^{2}(\Delta z) \\
& \\
&
\end{aligned}
$$

where:

- $K_{W \theta}$ is the noise band coefficient of the weight function $W_{\theta}(z)$ of the digital spatial spectral analysis,

- $m_{s}$ is the number of spectral-spatial counts of the signal group,

- $\mu_{I}$ is the signal to noise ratio at the input of DF channels of the AA, 
- $K_{\tau}(\Delta z)$ is the value of the normalized autocorrelation function for the weight function $W_{\theta}(z)$ of digital RP forming for the shift given by:

$$
K_{\tau}(\Delta z)=\frac{\sum_{z=0}^{Z-1} W_{\theta}(z) W_{\theta}(z-\Delta z)}{\sum_{z=0}^{Z-1} W_{\theta}^{2}(z)},
$$

- $\omega_{0} \in\left[\omega_{S . L} ; \omega_{S . H}\right]$ is the average frequency of passband of radio channels or the carrier frequency of the emission.

In view of Eq. (3) the general optimization conditions are defined as follows:

$$
\sigma_{\theta}^{2} \rightarrow \min
$$

While optimizing the researched DF method we have to define the type of the objective function and communication functions [15]. In order to do this based on Eqs. (2) and (3), we perform an analysis of the implementation of the optimized DF method. The analysis of Eq. (3) has shown that the dispersion $\sigma_{\theta}^{2}$ of direction estimation depends on the procedure of reconstructing the complex analytical signal within the AA aperture, and on correlation processing. In addition, the direction finder parameters, such as the number $Z$ of DF radio channels, the width $\Delta f_{k}$ of the passband of the radio channels and the sensitivity $\mu_{\mathrm{BX}}$, have significant limitations in the optimization process, with the capabilities of modern technology and implementation of direction finder's compactness and price-related requirements taken into consideration.

However, such parameters as $K_{W \theta}$ and $m_{S}$ are determined by the requirements for noise immunity of DF in a difficult EMS and by the provision of effective spatial selection of station interference and multipath interference. Therefore, their variation is also severely limited in order to optimize the accuracy of DF.

Therefore, the emission parameters of DF RES, such as the average or the carrier frequency $\omega_{0}$ of the time spectrum (obtained from the processing time) and the direction $\theta$ of arrival of radio waves are not affected in the algorithm of DF and have only a global limit concerning the range operating frequency range and the width of DF sector, respectively. The time $T_{a}$ during which radio emissions which are received at the same time within a passband $\Delta f_{k}$ of radio channels are analyzed, significantly affects interference immunity and the speed of DF's operation. However, analysis of time $T_{a}$ of the radio emission is not affected in the case of the general algorithm implementation and also in the reconstruction of a complex analytic signal.

Therefore, analysis of Eq. (3) shows that a significant impact on the dispersion $\sigma_{\theta}^{2}$ of direction estimation in constant devices and time expense is exerted by the spatial shift value $\Delta z$, as well as by the correlation coefficient $K_{W \theta}(\Delta z)$ of the weight function, which depends on the spatial shift $\Delta z$. Therefore, it is advisable to determine the objective function $F(\Delta z)$ for further optimization as a functional of variables $\Delta z$ and $K_{W \theta}(\Delta z)$ :

$$
F(\Delta z)=f\left[\Delta z, K_{W \theta}(\Delta z)\right]=\Delta z^{2} K_{W \theta}^{2}(\Delta z) .
$$

We perform a qualitative assessment of the features of the objective function $F(\Delta z)$. Analysis of Eq. (3) indicates that the spatial shift $\Delta z$ and the correlation coefficient $K_{W \theta}(\Delta z)$ of the weight function $W_{\theta}(z)$ are antagonistic parameters. The increase of the shift $\Delta z$ defines the essential decrease in the dispersion $\sigma_{\theta}^{2}$ of estimation of direction for back-quadratic dependence. In turn, the correlation coefficient $K_{W \theta}(\Delta z)$ decreases by increasing the value of the spatial shift $\Delta z$. The second variable of the objective function $K_{W \theta}(\Delta z)$ is the coefficient of correlation $K_{W \theta}(\Delta z)$, that is defined as [3]:

$$
K_{\tau}(\Delta z)=\frac{\sum_{z=0}^{Z-1} W_{\theta}(z) W_{\theta}(z-\Delta z)}{\sum_{z=0}^{Z-1} W_{\theta}^{2}(z)} .
$$

Analysis of Eq. (6) shows that the maximum value of the correlation coefficient $K_{W \theta}(\Delta z)$ is achieved at zero spatial shift $\Delta z=0: \max \left[K_{W \theta}(\Delta z)\right]=\left.1\right|_{\Delta z=0}$. By increasing the spatial shift $\Delta z$, the value of the correlation coefficient $K_{W \theta}(\Delta z)$ is reduced, which, in turn, determines an increase in the dispersion $\sigma_{\theta}^{2}$ of estimation of direction. Therefore, the task consists in finding the global extremum of the objective function $F(\Delta z)$. Taking into account the dependence between the value of the correlation coefficient $K_{W \theta}(\Delta z)$ and the spatial shift $\Delta z$, it is advisable to perform subsequent optimization, based on the scalar method, with an analysis of the relevant set of communication functions $f_{l}(X)[15]$.

\section{Optimization}

Considering Eqs. (3)-(6), the optimization equation relying on the objective function $F(\Delta z)$ and the optimum criterion, may be determined as:

$$
F(\Delta z)=\Delta z^{2} \frac{\sum_{z=0}^{Z-1} W_{\theta}(z) W_{\theta}(z-\Delta z)}{\sum_{z=0}^{Z-1} W_{\theta}^{2}(z)} \rightarrow \max
$$

The solution of Eq. 7 is the optimal value $\Delta z_{\mathrm{opt}}$ of the spatial shift $\Delta z$. Hence, the optimization of Eq. (7) takes the form of:

$$
\Delta z_{\mathrm{opt}}=\arg \max F(\Delta z) .
$$

We define the type of communication functions $f_{l}(X)$, the number $L$ and the type of the desired extremum of the objective function $F(\Delta z)$ for optimization.

At first, general spatial correlation processing must be carried out for one correlation processing cycle, using a single-channel correlator. Taking into consideration the 
first $f_{1}\left(N_{C}\right)$ and the second $f_{2}\left(N_{k}\right)$ the communication functions take the following form:

$$
\begin{aligned}
f_{1}\left(N_{C}\right) & =N_{C}=1, \\
f_{2}\left(N_{k}\right) & =N_{k}=1,
\end{aligned}
$$

where $N_{C}$ and $N_{k}$ are the numbers of cycles and apparatus channels used in correlation processing respectively.

Secondly, a reconstructed spatial analytic signal $U_{k . S}(\mathrm{j} z)$ is used as a limited sum of spatial harmonic components. Its spatial frequencies $\Omega_{p}$ are the result of FFT properties. They are multiples of the base frequency $\Omega_{1}$, whose period is equal to the value $d z$ of AA aperture [16]:

$$
U_{k . S}(\mathrm{j} z)=\sum_{p=p_{L}}^{p_{H}} U_{k . S}\left(\Omega_{p}\right) e^{\mathrm{j} \Omega_{p} z+\Psi_{p}},
$$

where $p_{H}=p_{L}=m_{S}$ is the dimension of the signal group and $U_{k . S}\left(\Omega_{p}\right), \Psi_{p}$ are the module and the argument of the $p$-th component of the signal group. Equation 10 determines the periodicity of distribution of counts of the spatial analytical signal $U_{k . S}(\mathrm{j} z)$ with a period equal to the value $d z$ of the AA aperture:

$$
U_{k . S}((\mathrm{j} z))=U_{k . S}[\mathrm{j}(z \pm d Z m)]
$$

where $m=1,2, \ldots, Z-1$.

Let's also note that the weight function $W_{\theta}(z)$ of the digital spatial spectral analysis has the mirror-symmetric distribution of counts within the AA aperture, concerning its center coordinates $\frac{d Z}{2}$.

Therefore, an unambiguous value of the correlation coefficient $K_{W \theta}(\Delta z)$ will be formed by using a spatial shift $\Delta z$, that does not exceed in terms of value $\frac{d Z}{2}$, the AA aperture. The domain of the correlation coefficient $K_{W \theta}(\Delta z)$ and the objective function $F(\Delta z)$ will be $[-0.5 d Z ; 1.5 d Z]$. As a result, the third $f_{3}(\Delta z)$ and the fourth $f_{4}(z)$ communication functions have the form:

$$
\begin{aligned}
f_{3}(\Delta z) & =\frac{d Z}{2}>\Delta z>-\frac{d Z}{2}, \\
f_{4}(z) & =d(Z-1)>z \geq 0 .
\end{aligned}
$$

In the third step, as spatial reconstruction of the analytical signal $U_{k . S}(\mathrm{j} z)$ is realized based on of the FFT algorithm, its spatial counts will coincide with the spatial counts $U_{z}\left(t_{k}\right)$ of received mixtures within the AA aperture only at $m d$ locations of the AA antenna elements. In other locations of AA, the approximation error may be significant [16]. Considering this, it is appropriate to use only the discrete spatial shift which is a multiple of the AA step $d$. As a result, the corresponding value of the fifth communication function $f_{5}(\Delta z)$ is as follows:

$$
f_{5}(\Delta z)=\Delta z=m d
$$

Let's perform a probabilistic analysis of the features of the spatial distribution of counts of the analytical signal $U_{k . S}(\mathrm{j} z)$ that should be presented as an additive mixture of signal $S_{a S}(\mathrm{j} z)$ and noise $S_{a N}(\mathrm{j} z)$ components within the AA aperture:

$$
U_{k . S}(\mathrm{j} z)=S_{a S}(\mathrm{j} z)+S_{a N}(\mathrm{j} z) \text {. }
$$

The noise component $S_{a N}(\mathrm{j} z)$ is formed as the sum of $m_{S}$ harmonic noise components $S_{a N . m}(\mathrm{j} z)$ of the signal group, that is:

$$
S_{a N}(\mathrm{j} z)=\sum_{m=1}^{m_{S}} S_{a N \cdot m}(\mathrm{j} z) .
$$

Every harmonic noise component $S_{a N . m}(\mathrm{j} z)$ is a narrowband random process with a normal distribution of probability density of counts. As a result, the noise component $S_{a N}(\mathrm{j} z)$ of the spatial analytical signal $U_{k . S}(\mathrm{j} z)$ is also a normal narrowband random process, and its power $P_{a} N$ will be equal to the sum of powers $P_{a N . m}$ of $m_{S}$ constituent components:

$$
P_{a N}=\sum_{m=1}^{m_{S}} P_{a N . m}
$$

In addition, the noise component $S_{a N}(\mathrm{j} z)$ of the spatial analytical signal $U_{k . S}$ (jz has a correlation interval $\Delta z_{\text {cor }}$, which is determined by its bandwidth $\frac{m_{S}-1}{d z}$ as [11]:

$$
\Delta z_{\mathrm{cor}}=\frac{d z}{m_{S}-1} .
$$

The analysis of Eq. (17) shows that it is necessary for the spatial shift $\Delta z$ not to be less than the correlation interval $\Delta z_{\text {cor }}$ to ensure effective statistical estimation of the difference $\Delta \hat{\Psi}_{A k}\left(\Delta z, \Omega_{p}\right)$ between the arguments of the signal component $S_{a} S(\mathrm{j} z)$ of the spatial analytical $U_{k . S}(\mathrm{j} z)[11]$. Taking this into account, the sixth communication function $f_{6}(\Delta z)$ takes the form:

$$
f_{6}(\Delta z)=\Delta z \geq \frac{d z}{m_{S}-1} .
$$

It can be concluded from Eqs. (9)-(18) that optimization should be carried out taking into account the six $L=6$ functions of communication and the search for a global constrained extremum type maximum.

Considering the optimum value of the spatial shift $\Delta z_{\text {opt }}$, Eq. (8) is defined as the solution of the corresponding differential equation:

$$
\Delta z_{\mathrm{opt}}=\arg \frac{\partial F(\Delta z)}{\partial \Delta z}=0
$$

We take into account that, in general, the weight function $W_{\theta}(z)$ is nonlinear. Therefore, it is advisable to use nonlinear programming methods to solve the optimization problem [15].

The Mathcad software package was used under the following conditions. The number of DF channels $Z=64$, the types of weight functions are minimum 3-sample Blackman-Harris and minimum 4-sample Blackman-Harris - for the solution of Eq. (19).

The advantage of such an approach is that they are optimized by the minimum of the area of all side lobes and have 
a sufficiently low level of maximum side lobes $(-67 \mathrm{~dB}$ and $-92 \mathrm{~dB}$, accordingly). Additionally, the windows show a decrease in the level of side lobes $-6 \mathrm{~dB} /$ oct, as opposed to the Dolph-Chebyshev window, for which the decrease in the level of side lobes is $-6 \mathrm{~dB} /$ oct [17]. Consequently,

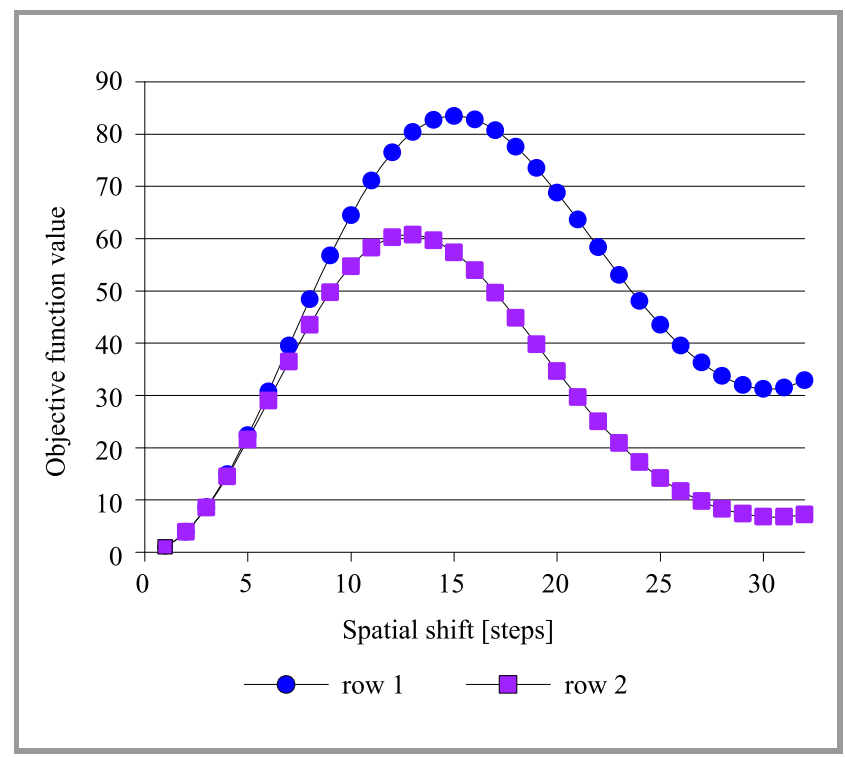

Fig. 2. Spatial shift optimization results.

the use of these windows provides an efficient selection of powerful random interference that has the uniform and the Gaussian distribution of the probability density in the direction of arrival. Such a version of EMS is typical for conducting of radio monitoring in the urban conditions in the presence of networks of comprised of moving sources of radio emission sources. The minimum 3-sample BlackmanHarris weight function $W_{\theta}(z)$ has a $-67 \mathrm{~dB}$ level of side lobes and is defined by [16]:

$$
\begin{aligned}
W_{\theta}(z)=0.42329+0.49755 \cos & \frac{2 \pi z}{Z} \\
& +0.07922 \cos \frac{4 \pi z}{Z} .
\end{aligned}
$$

The minimum 4-sample Blackman-Harris weight function has $92 \mathrm{~dB}$ level of side lobes and is defined by [16]:

$$
\begin{aligned}
W_{\theta}(z)=0.35875 & -0.48829 \cos \frac{2 \pi z}{Z} \\
& +0.14128 \cos \frac{4 \pi z}{Z}-0.01168 \cos \frac{2 \pi z}{Z} .
\end{aligned}
$$

Optimization results for the minimum 3-sample BlackmanHarris window $W_{\theta}(z)$ (row 1) and for the minimum 4-sample Blackman-Harris window $W_{\theta}(z)$ (row 2) are shown in Fig. 2.

Analysis of Fig. 2 shows that the objective function $F(\Delta z)$ for both types of windows has a bell-shaped form with one global maximum. The maximum value of the objective function $F(\Delta z)$ will be achieved when $\Delta z_{\text {opt }}=15$ for the minimum 3-sample Blackman-Harris window, and
$\Delta z_{\mathrm{opt}}=13$ for the minimum 4-sample Blackman-Harris window.

Therefore, the purpose of this article that is concerned with the optimization problem of direct digital method of correlative-interferometric radio DF with two-dimensional correlative processing of a reconstructed complex spatial signal and with determination of the optimal spatial shift has been achieved.

\section{Simulation Results}

A simulation of the direction finder was conducted [3]. A software model was developed in Mathcad for the following initial conditions:

- signal type - continuous with linear frequency modulation,

- width of signal $\Delta f_{S}=0.6 \mathrm{MHz}$,

- passband of DF radio channels $\Delta f_{k}=10 \mathrm{MHz}$,

- working frequency of the signal $f_{S}=2 \mathrm{GHz}$,

- sampling frequency $f_{d}=2 \Delta f_{k}=20 \mathrm{MHz}$,

- numbers of signal time counts analyzed $N_{S}=2048$,

- duration of analysis $T_{a}=0.1 \mathrm{~ms}$,

- AA type - linear AA with the number of DF reception channels $Z=64$,

- given direction towards RES $\theta=60^{\circ}$ in relation to the AA aperture line,

- AA step size $d=0.5 \mathrm{~m}$,

- number of tests to assess the middle square deviation (MSD) of estimation of direction $=50$,

- number of counts of signal groups $m_{S}=6$ for the minimum 3-sample Blackman-Harris window $W_{\theta}(z)$,

- number of counts of signal groups $m_{S}=8$ for the minimum 4-sample Blackman-Harris window $W_{\theta}(z)$.

As a result, the simulations performed for the analyzed DF method we receive a range of dependencies concerning MSD of direction estimation, obtained based on the $\Delta z$ spatial shift values (in AA steps of $d$ ) for the signal-tonoise ratio of $0 \mathrm{~dB}$ for the minimum 3-sample and 4-sample Blackman-Harris windows, as shown in Fig. 3.

Figure 3 presents two curves: row 1 - for the minimum 3-sample Blackman-Harris window $W_{\theta}(z)$, and row 2 - for the minimum 4-sample Blackman-Harris window $W_{\theta}(z)$.

Figure 3 shows that the value of MSD is a smooth line with one global minimum. The minimum MSD value is equal to $\sigma_{\theta}=0.02$, when $\Delta z=15$, row 1 and $\sigma_{\theta}=0.025$, when $\sigma_{\theta}=0.025$, row 2. The lower value of MSD of direction estimation is obtained by using the BlackmanHarris 3-sample window (row 1). This is consistent with 
the theory, as this window has a smaller equivalent noise bandwidth [17].

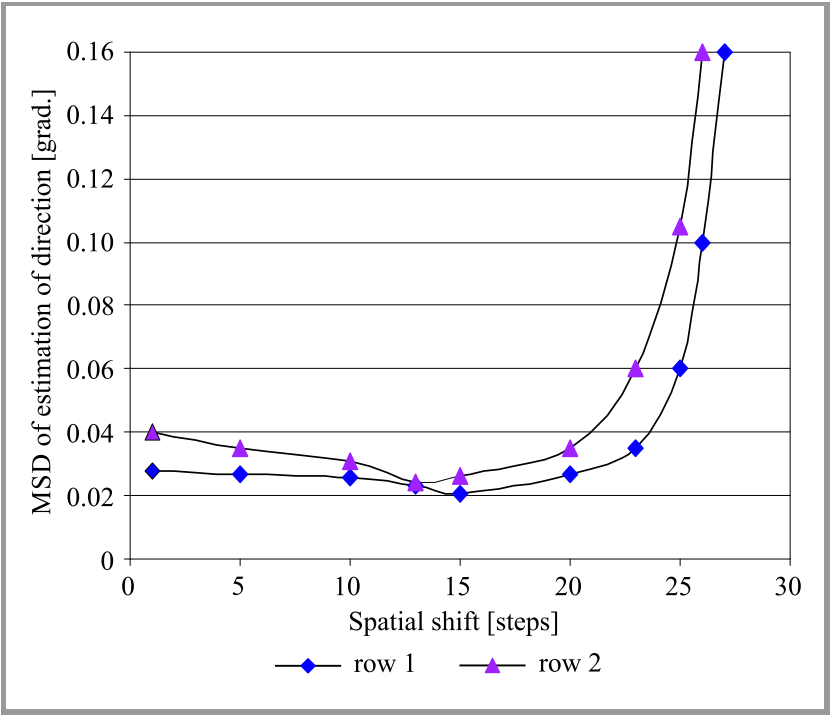

Fig. 3. Range of dependencies between MSD of direction estimation and spatial shift values.

Let's evaluate the effect of optimization on the accuracy of DF. The experiments have shown that within the range of $\Delta z$ changes from 1 to 32 , the value of MSD of direction estimation varies within $[0.02 ; 1]$ degrees, that is by 50 times. This proves that a significant impact is exerted on the accuracy of the optimization process. With the use of certain optimal spatial shift values of $\Delta z=13$ and $\Delta z=15$, it is possible to improve the accuracy of direction estimation of RES or to increase the speed of the system by significantly reducing the duration of radio frequency analysis.

The received dependence shown in Fig. 3 can be conventionally divided into three ranges of $\Delta z$ values. For example, for row 2 in the first range $[1 ; 13]$, the value of MSD of direction estimation decreases gradually by almost 2 times, from $\sigma_{\theta}=0.04$ to $\sigma_{\theta}=0.025$. In the second range of $\Delta z=[14 ; 22]$, the value of MSD of direction estimation increases gradually to $\sigma_{\theta}=0.04$. In the third range of values $\Delta z=[23 ; 32]$, the MSD of direction estimation increases rapidly with the quadratic dependence. The obtained results confirm the high level of accuracy of the DF method investigated, at a low signal-to-noise ratio of $0 \mathrm{~dB}$.

The comparative analysis of Figs. 2, 3 shows that the analytical results of optimization and the simulation results are the same for spatial shift values $\Delta z$ for which the MSD of direction estimation is minimal. Therefore, the aim of the article is achieved.

\section{Conclusions and Future Work}

In this article, the main optimized parameter of the correlative-interferometric method of direction finding with two-dimensional correlative processing of spatial signal in the aperture of a linear antenna array is determined. It is the $\Delta z$ value of the spatial shift within the AA aperture for which the phase shift and the frequency of spatial analytical signal are estimated.

Analytical optimization of the parameters of the studied method has shown that the maximum value of the objective function $F(\Delta z)$ with the optimal spatial shift $F(\Delta z)$ for the minimum 3-sample Blackman-Harris window $W_{\theta}(z)$ and $W_{\theta}(z)$ for the minimum 4-sample Blackman-Harris window $W_{\theta}(z)$ is achieved.

Analysis of the simulation results has shown that the value of MSD of direction estimation is monotonically dependent on the spatial shift $\Delta z$ with one minimum, and equals $\sigma_{\theta}=0.02$ for the spatial shift of $\Delta z=15$ for the minimum 3-sample Blackman-Harris window $W_{\theta}(z)$, and equals $W_{\theta}(z)$ for the spatial shift of $W_{\theta}(z)$ for the minimum 4-sample Blackman-Harris window $W_{\theta}(z)$. The lower value of MSD of direction estimation is obtained by using the Blackman-Harris 3-sample window. This is consistent with theory, because this window has a smaller equivalent noise bandwidth.

The analytical results of optimization and the simulation results are the same for both windows. The value of MSD will be minimal and is equal $\sigma_{\theta}=0.02$ for the spatial shift of $\Delta z=15$ while using the minimum 3-sample BlackmanHarris window the side lobes level of $-67 \mathrm{~dB}$, which offers a high degree of interference immunity of DF.

The results obtained are suitable for application in the operation of radio monitoring, telecommunications and radio navigation systems which function in a complex dynamic EMS.

\section{References}

[1] G. Kratschmer (Ed.), Introduction into Theory of Direction Finding. Radiomonitoring and Radiolocation 2010/2011. Munich: Rohde \& Schwarz GmbH \& Co. HW - UKD, 2011, pp. 85-101.

[2] A. M. Rembovskiy, A. V. Ashyhmin, and V. A. Kuzmin, Radiomonitoring - tasks, methods, devices - 2nd ed. Hotline - Telecom, 2010.

[3] V. V. Tsyporenko and V. G. Tsyporenko, "Development of direct method of direction finding with two-dimensional correlative processing of spatial signal", Eastern-Europ. J. of Enterpr. Technologies vol. 6 , no. 84 , pp. $63-70,2016$ (doi: $10.15587 / 1729-4061.2016 .85599)$.

[4] J.-H. Lee and J.-M. Woo, "Interferometer direction finding system with improved DF accuracy using two different array configurations", IEEE Anten. and Wirel. Propag. Let., vol. 14, pp. 719-722, 2014 (doi: 10.1109/LAWP.2014.2377291).

[5] J. Yang, W. Chen, L. Li, and X. Ni, "Long baseline direction finding and localization algorithms for noise radiation source", in Proc. 12th Int. Conf. on Signal Process. ICSP 2014, Hangzhou, Zhejiang, China, 2014, pp. 52-57 (doi: 10.1109/ICOSP.2014.7014968).

[6] H. Gazzah, J. P. Delmas, and S. M. J. Larsys, "Direction-finding arrays of directional sensors for randomly located sources", IEEE Transac. on Aerospace and Elec. Sys., vol. 52, no. 4, pp. 1995-2003, 2016 (doi: 10.1109/TAES.2016.150655).

[7] S. Van Doan, J. Vesely, P. Janu, P. Hubacek, and X. L. Tran, "Optimized algorithm for solving phase interferometer ambiguity", in Proc. 17th Int. Radar Symp. IRS 2016, Kraków, Poland, 2016, pp. 1-6 (doi: 10.1109/IRS.2016.7497353). 
[8] X. Fu, N. D. Sidiropoulos, W.-K. Ma, and J. Tranter, "Blind spectra separation and direction finding for cognitive radio using temporal correlation-domain ESPRIT", in Proc. 39th Int. Conf. on Acoustics, Speech and Signal Proces. ICASSP 2014, Florence, Tuscany, Italy, 2014, pp. 7749-7753 (doi: 10.1109/ICASSP.2014.6855108).

[9] K. V. Rangarao and S. Venkatanarasimhan, "Gold-MUSIC: A variation on MUSIC to accurately determine peaks of the spectrum", IEEE Transac. on Anten. and Propag., vol. 61, no. 4, pp. 2263-2268, 2013 (doi: 10.1109/TAP.2012.2232893).

[10] W. Chen, X. Xu, S. Wen, and Z. Cao, "Super-resolution direction finding with far-separated subarrays using virtual array elements", IET Radar, Sonar \& Navigation, vol. 5, no. 8, pp. 824-834, 2011 (doi: 10.1049/iet-rsn.2010.0289).

[11] V. V. Karavajev and V. V. Sazonov, Statistic theory of passive location. Moscow: Radio and Communication, 1987 [in Russian].

[12] Q. Cui, J. Wang, J. Han, and X. Tao, "Pattern optimisation algorithm for vertical cell splitting system with active antenna arrays", IET Elec. Let., vol. 50, no. 20, pp. 1416-1417, 2014 (doi: 10.1049/el.2014.2023).

[13] J.-H., Lee, J.-H. Lee, and J.-M. Woo, "Method for obtaining threeand four-element array spacing for interferometer direction-finding system", IEEE Anten. and Wirel. Propag. Let., vol. 15, pp. 897-900, 2016 (doi: 10.1109/LAWP.2015.2479224).

[14] T. Sánchez, C. Gómez, G. I. Angel Castillo, and M. Patiño Bernal, "Radio direction finding system for spectrum management activities in developing countries", in Proc. 17th Int. Symp. on Anten. and Propag. APSURSI 2016, Fajardo, Puerto Rico, 2016, pp. 1663-1664 (doi: 10.1109/APS.2016.7696538).

[15] G. Fornarelli and L. Mescia, Swarm Intelligence for Electric and Electronic Engineering. Hershey, PA, USA: IGI Global, 2012 (ISBN: 9781466626669).

[16] J. G. Proakis and D. G. Manolakis, Digital Signal Processing, Principles, Algorithms, and Applications, 4th Edition. Upper Saddle River, NJ, USA: Prentice-Hall Inc., 2006 (ISBN: 9780131873742).

[17] F. J. Harris, "On the use of windows for harmonic analysis with the discrete Fourier transform", Proc. of the IEEE, vol. 66, no. 1, pp. 51-83, 1978 (doi: 10.1109/PROC.1978.10837).

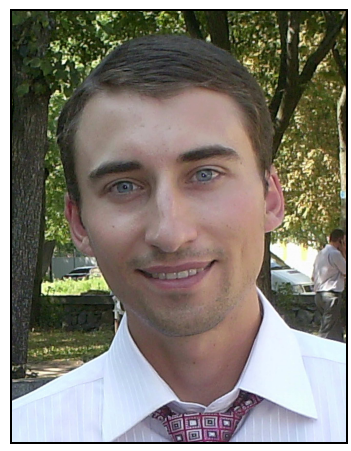

Vitaliy Tsyporenko obtained his Ph.D. in radio-technical and television systems in 2011, with the thesis focusing on researching new direct digital correlation-interferometric DF methods. At present, he works as an associate professor at the Zhytomyr State Technological University (ZSTU), Ukraine. His current research interests focus on correlation-interferometric direction finding.

(iD) https://orcid.org/0000-0001-8559-006X

E-mail: tsyporenko.1985@gmail.com

Zhytomyr State Technological University

Zhytomyr, Ukraine

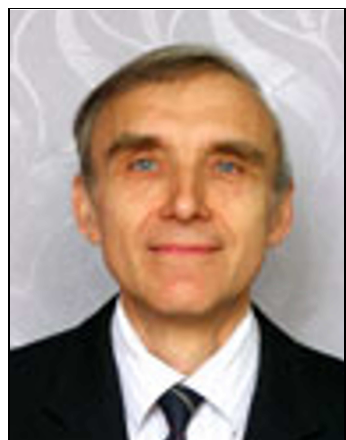

Valentyn Tsyporenko obtained his $\mathrm{Ph} . \mathrm{D}$. in radio-technical systems in 1995, with the thesis focusing on researching new digital signal processing methods. At present, he holds the position of an associate professor at ZSTU, Ukraine. His current research activities focus on direction finding.

https://orcid.org/0000-0002-6843-8960

E-mail: tsyporenkovg@gmail.com

Zhytomyr State Technological University

Zhytomyr, Ukraine 